\title{
La medición multidimensional de la pobreza a nivel intraurbano en Ciudad Juárez, Chihuahua (2012)
}

\author{
The multidimensional measure of poverty at the \\ intraurban level in Ciudad Juarez, Chihuahua (2012)
}

\author{
César Mario Fuentes ${ }^{\text {a* }}$ (i) https://orcid.org/0000-0002-7224-5723 \\ Sergio Peña ${ }^{a}$ (iD) https://orcid.org/0000-0002-9505-4057 \\ Vladimir Hernández ${ }^{b}$ (i) https://orcid.org/0000-0003-0206-9768
}

Recibido el 21 de septiembre de 2016.

Aceptado el 20 de septiembre de 2017.

${ }^{*}$ Autor para correspondencia: César Mario Fuentes, correo electrónico: cfuentes@colef.mx

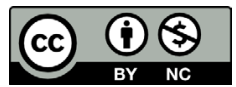

Esta obra está protegida bajo una Licencia Creative Commons Atribución-NoComercial 4.0 Internacional.

\begin{abstract}
${ }^{a}$ El Colegio de la Frontera Norte, Departamento de Estudios Urbanos y del Medio Ambiente en la sede de Ciudad Juárez, Chihuahua, México, correos electrónicos: cfuentes@colef.mx, spena@colef.mx

b Universidad Autónoma de Ciudad Juárez, Departamento de Arquitectura, Chihuahua, México, correo electrónico: vladimir.hernandez@uacj.mx
\end{abstract}

\section{Resumen}

El objetivo del artículo es realizar la medición multidimensional de la pobreza a nivel intraurbano en Ciudad Juárez, Chihuahua en el año 2012. La metodología que aplicó fue la misma que desarrolló Coneval en 2010 para su medición multidimensional de la pobreza a nivel nacional, estatal y municipal. Para poder aplicar la metodología de Coneval a nivel intraurbano se propuso como alternativa la construcción de las unidades de análisis territorial (UTA) mediante una técnica de análisis multivariado. Los resultados permiten identificar las UTA de la ciudad en donde la pobreza se padece con una mayor incidencia, intensidad y profundidad, lo cual es una poderosa herramienta para el diseño de políticas públicas focalizadas en el territorio. Las áreas de la ciudad que se localizan en el poniente y suroriente presentan los mayores rezagos sociales. Una de las principales limitaciones del estudio fue la imposibilidad financiera de ampliar el levantamiento de la encuesta con el objetivo de mejorar la calidad de la estimación.

Palabras clave: pobreza multidimensional, Coneval, Ciudad Juárez.

\section{Abstract}

The aim of this article is to measure multidimensional poverty at the intra-urban level in Ciudad Juárez, Chihuahua, in 2012. The methodology used was the same as that developed by Coneval in 2010 for its multidimensional measurement of poverty at the national, state and municipal levels. Territorial units of analysis (TUAs) were developed using a multivariate analysis technique to apply the methodology created by Coneval at the intra-urban level. The results make it

CÓMO CITAR: Fuentes, C. M., Peña, S. y Hernández, V. (2018). La medición multidimensional de la pobreza a nivel intraurbano en Ciudad Juárez, Chihuahua, [The multidimensional measure of poverty at the intraurban level in Ciudad Juarez, Chihuahua]. Estudios Fronterizos, 19, e001. doi:10.21670/ref.1801001 
possible to identify the TUAs in the city with greater incidence, intensity and depth of poverty. This method represents a powerful tool to design targeted public policies in the territory. Western and southeastern parts of the city exhibit greater social need. One of the main limitations of the study was the financial impossibility of expanding the survey implementation to improve the quality of the estimates.

Keywords: multidimensional poverty, Coneval, Ciudad Juárez.

\section{Introducción}

En México a partir del año 2008 se lleva a cabo la medición de la pobreza desde un enfoque multidimensional en cumplimiento de uno de los compromisos plasmados en la Ley General de Desarrollo Social (LgDs). Para cumplir con el mandato, el Instituto Nacional de Estadística, Geografía e Informática (Inegi) y el Consejo Nacional de Evaluación de la Política de Desarrollo Social (Coneval) incluyeron el Módulo de Condiciones Socioeconómicas (mCS) como un anexo a la Encuesta Nacional de Ingresos y Gastos de los Hogares (Enigh) para generar la información estadística para realizar la medición de la pobreza multidimensional (Garza-Rodríguez, 2016). Sin embargo, debido a la representatividad estadística de la encuesta, la medición solo es estimada a escala nacional, estatal y municipal pero no a nivel intraurbano, lo cual dificulta la focalización territorial de los programas sociales a escala urbana. Por lo anterior, en México recientemente algunos gobiernos locales como el de la Ciudad de México y el de Ciudad Juárez realizaron esfuerzos importantes para poder generar sus propias bases de datos con la finalidad de hacer sus mediciones de la pobreza multidimensional a nivel intraurbano. Lo anterior, con el objetivo de poder focalizar de manera territorial los programas sociales, evaluarlos y replicarlos en el tiempo (Sánchez, Boltivinik, Ruiz y Figueroa, 2011).

En el caso de Ciudad Juárez debido a los altos costos sociales de la violencia homicida y la pérdida de miles de empleos producto de la recesión económica global, entre 2008 y 2010 se incrementó el número de personas en situación de pobreza (Coneval, 2010). Lo anterior, se tradujo en un aumento de la cantidad de personas que solicitaban apoyos en especie de los distintos programas sociales que ofrece el gobierno municipal para combatir la pobreza. Pero al no contar con recursos suficientes para hacer frente a la contingencia optaron por solicitar apoyos económicos adicionales a otros niveles de gobierno.

Con el fin de justificar el incremento de los recursos solicitados era necesario hacer una nueva medición de la pobreza que fuera compatible con la metodología "oficial". Ya que en México el resultado de la medición "oficial" de la pobreza se constituye como uno de los criterios para la designación de los recursos del gobierno federal asignados a los programas sociales. Además, con el propósito de hacer un uso más eficiente de los recursos escasos se decidió realizar la medición de la pobreza multidimensional a nivel intraurbano para mejorar la focalización territorial de los programas sociales.

En este contexto, el objetivo del artículo es realizar la medición de la incidencia, intensidad y profundidad de la pobreza multidimensional a nivel intraurbano en Ciudad Juárez, Chihuahua en el año 2012.

La estructura del artículo corresponde íntegramente al objetivo que fue planteado. La primera sección presenta los distintos métodos de medición de la pobreza que se 
han aplicado en México, así como una revisión de los trabajos en los cuales se reportan mediciones de la pobreza a nivel regional o urbano. La segunda sección muestra la metodología usada para la estimación de la pobreza multidimensional a nivel intraurbano. La tercera sección describe el desempeño de los indicadores de pobreza en las ciudades de la frontera norte y en específico el caso de Ciudad Juárez y la medición de la pobreza a nivel municipal. La cuarta parte reporta los resultados de la incidencia, intensidad y profundidad de la pobreza multidimensional a nivel intraurbano en Ciudad Juárez, Chihuahua. Finalmente, se presentan las conclusiones.

\section{Los métodos para la medición de la pobreza y su medición a nivel regional e intraurbano}

\section{Métodos para la medición de la pobreza en América Latina}

La pobreza tiene varias definiciones dependiendo del contexto socioeconómico, demográfico o cultural en que se use, por ejemplo, el Banco Mundial (1990, p. 26) la define como "un nivel de vida mínimo". Barneche et al., (2010, p. 31) consideran que en términos generales, "la pobreza es entendida como la situación de privación que obliga a quienes la padecen a llevar una vida por fuera de los estándares socialmente establecidos". Pobreza y exclusión social son conceptos estrechamente relacionados, el primero en su acepción contemporánea puede equipararse al segundo como un proceso dinámico y acumulativo que separa a las personas de los recursos necesarios para acceder a las condiciones materiales de existencia típica de una sociedad históricamente determinada. Recientemente la definición que más es usada en América Latina para la identificación de la pobreza se centra en "las acepciones concernientes a necesidad, estándar de vida, o insuficiencia de recursos. Así, los indicadores más utilizados atienden a la satisfacción de ciertas necesidades, al consumo de bienes o el ingreso disponible" (Barneche et al., 2010, p. 31).

El concepto de pobreza, para su medición, debe de cumplir con dos condiciones: 1) la identificación y 2) la agregación. La primera se refiere al registro de un grupo de individuos en la categoría de pobres y la segunda "permite reunir a los pobres en una imagen global de pobreza” (Caloca y Ortiz, 2016, p. 102).

En este sentido, en la literatura especializada sobre pobreza se señala que existen diversos métodos para su identificación. Boltvinik (2003) propuso una tipología al clasificar en un primer grupoa los métodos indirectos, no normativosy unidimensionales como las líneas de pobreza (LP). En un segundo grupo a los métodos indirectos ${ }^{1}$ semi- $^{2}$ normativos y normativos y unidimensionales ${ }^{2}$ como la canasta normativa alimentaria (CNA), canasta normativa generalizada (N) y las líneas de la pobreza subjetivas. En un tercer grupo a los métodos directos, normativos y multidimensionales como el

\footnotetext{
${ }^{1}$ La medición indirecta evalúa si los recursos (por lo general reducidos al ingreso corriente) del hogar son suficientes para satisfacer las necesidades, independientemente de si esto ocurre o no. Otra manera de distinguir la medición directa de la indirecta es llamándole fáctica a la directa y potencial a la indirecta (Boltvinik, 2003).

${ }^{2}$ Lo unidimensional de los métodos se refieren a una sola dimensión y multidimensional a varias dimensiones del bienestar (Boltvinik, 2003).
} 
de necesidades básicas insatisfechas (NBI), índice de privación y carencia forzada de satisfactores básicos socialmente percibidos (CFSBSP). Finalmente, en un cuarto grupo a los métodos combinados, semi-normativos y normativos ${ }^{3}$ y multidimensionales como la línea de la pobreza objetiva, métodos de medición integrada de la pobreza (MMIP) y la medición con la metodología de Coneval.

En este trabajo no se pretende hacer una revisión exhaustiva de todos los métodos de medición de la pobreza sino solo presentar los que se han aplicado en América Latina. En México hasta el año 2006 la medición oficial de la pobreza se llevó a cabo desde una perspectiva unidimensional, en la que se utiliza al ingreso como una variable de aproximación del bienestar económico de la población. En dicha medición se suele definir un umbral o línea de pobreza (que representa el ingreso mínimo necesario para adquirir una canasta de bienes considerados indispensables) y se compara con el ingreso de los hogares para identificar a la población que carece de las condiciones necesarias para satisfacer sus necesidades, siempre y cuando se puedan adquirir a través de los mercados de bienes y servicios.

\section{Métodos indirectos}

El método de la línea de pobreza (LP) utiliza el ingreso o el gasto de consumo como medida del bienestar, estableciéndose el valor per cápita de una canasta mínima de consumo necesario para la sobrevivencia. "La línea de pobreza extrema corresponde al valor per cápita de la canasta que contiene sólo rubros alimenticios. La línea de pobreza total comprende el valor per cápita de los rubros alimenticios y no alimenticios" (Barneche et al., 2010, pp. 32-33). En este método son considerados pobres extremos quienes tienen ingresos o gastos per cápita por debajo del valor de la canasta mínima alimentaria. De igual manera son:

Pobres no extremos quienes tienen ingresos o gastos per cápita por arriba de la línea de pobreza extrema, pero por debajo de la línea de pobreza total. Finalmente, se consideran no pobres a quienes tienen ingresos o gastos per cápita por arriba de la línea de pobreza total (Barneche et al., 2010, pp. 32-33).

\section{Métodos directos normativos}

Método de las necesidades básicas insatisfechas (NBI). Este método es directo y es usado para la identificación y medición de la pobreza en América Latina a partir de inicios de la década de los ochenta (Feres y Mancero, 2000). El cual toma en consideración un "conjunto de indicadores relacionados con necesidades básicas estructurales (vivienda, educación, salud, infraestructura pública, y otros) que se requiere para evaluar el bienestar individual" (Barneche et al., 2010, p. 33).

\footnotetext{
${ }^{3}$ Los métodos normativos se entienden como aquellos que definen el umbral que separa a los pobres de los no pobres con base en una noción de nivel mínimo de vida aceptable o en una noción de necesidades básicas que se deben satisfacer y los satisfactores requeridos para que esta condición se cumpla (Boltvinik, 2003).
} 
A pesar de que cada país posee características propias en torno al fenómeno de la pobreza, existen carencias comunes (universales) establecidas para la aplicación del método entre las cuales figuran: el hacinamiento, la vivienda inadecuada (condiciones de habitabilidad), el abastecimiento inadecuado de agua, la carencia o inconveniencia de servicios sanitarios y la eliminación de excretas, y la inasistencia de los menores en edad escolar a escuelas primarias son indicadores indirectos de capacidad económica.

En México las principales críticas que se le hicieron a los métodos unidimensionales como la LP y el de NBI se centraron en la necesidad de ampliar las fuentes de bienestar (Boltvinik, 1990a; 1990b; 1992). En el primer caso, solo se integra el ingreso, como si la satisfacción de necesidades únicamente dependiera del consumo privado corriente de los hogares. En el segundo caso, la medición se centra en indicadores de satisfactores que dependen de la propiedad de activos de consumo (vivienda) o de los derechos de acceso a servicios públicos (agua, drenaje, educación) y no considera las demás fuentes de bienestar (Boltvinik, 1992). Por ello se hizo necesario desarrollar otras mediciones que tomaran en cuenta la multidimensionalidad del fenómeno de la pobreza.

\section{Métodos combinados seminormativos}

En México se han usado diversos métodos para la medición de la pobreza multidimensional entre los que destacan el Método de Medición Integrada de la Pobreza (мmip), el cual desde inicios de la década de los noventa fue propuesto por Boltvinik (1992), aunque originalmente había sido desarrollado por Beccaria y Minujin (1987) y recientemente la medición oficial de la pobreza multidimensional realizada por Coneval (Coneval, 2009).

El MMIP fue desarrollado por Boltvinik (1992), con el propósito de superar las limitaciones de los métodos de las LP y NBI. Boltvinik (1992) considera que la pobreza es un fenómeno multidimensional en que el bienestar de los hogares depende de cinco fuentes adicionales al ingreso corriente, base del enfoque indirecto: a) los activos no básicos y la "capacidad" de endeudamiento del hogar; b) el patrimonio familiar; c) el acceso a bienes y servicios gratuitos (derechos sociales); $d$ ) tiempo libre y disponible para el trabajo doméstico, la educación y el reposo; y $e$ ) nivel de educación, relacionado con los conocimientos de las personas. Para Boltvinik (1992) una aproximación al fenómeno de la pobreza solo es posible si se toman en cuenta todas estas dimensiones.

Medición de la pobreza multidimensional (Coneval). Esta metodología de medición de la pobreza adoptada por el Coneval ${ }^{4}$ se basa en el uso de los recursos monetarios de los hogares como indicador indirecto del bienestar (Comité Técnico para la Medición de la Pobreza [CTMP], 2002). Retoma propuestas metodológicas que combinan mediciones de pobreza por ingresos con las de necesidades básicas insatisfechas (Beccaria y Minujin, 1987; Feres y Mancero, 2000; Gordon, 2006). Además, la metodología adoptada por el Coneval estableció una serie de criterios con el fin de cumplir con los principios de transparencia, rigor técnico e imparcialidad, así mismo de ser una herramienta útil en la arena de las políticas públicas del desarrollo social (Coneval, 2011).

\footnotetext{
${ }^{4}$ En esta sección no se busca hacer una revisión detallada de la metodología aplicada por el Coneval.
} Para una revisión exhaustiva consúltese en Inegi (2011). 
De igual manera, el Coneval busca que la metodología de medición satisfaga el criterio de cumplir con los ordenamientos de la ley de desarrollo social y disposiciones normativas aplicables, con el objetivo de que se pueda identificar a la población en situación de pobreza (Coneval, 2011).

La identificación de la población en situación de pobreza es resultado del diagnóstico de su situación económica y de las carencias sociales que padece. Para una persona la condición de carencia social puede darse como consecuencia de una o más de las privaciones siguientes: 1) rezago educativo, 2) falta de acceso a los servicios de salud, 3) falta de acceso a la seguridad social, 4) vivienda de calidad inadecuada o de espacios insuficientes, 5) indisponibilidad de algún servicio básico en la vivienda, y 6) falta de acceso a la alimentación.

De esta forma, para la identificación de los pobres multidimensionales se utiliza el criterio de quiénes presentan al menos una carencia social y cuyos ingresos son inferiores a la línea de bienestar económico (LBE). Esta última especifica el ingreso necesario para adquirir las canastas alimentaria y no alimentaria de bienes y servicios. Dentro del grupo de personas en pobreza multidimensional, se identifican a las personas en pobreza extrema y en pobreza moderada. Las primeras son aquellas cuyo ingreso total es insuficiente incluso para comprar la canasta que permite satisfacer sus necesidades alimentarias y que, además, tienen tres o más carencias sociales. Las segundas son aquellas personas con un ingreso por debajo de la línea de bienestar económico y tienen una o dos carencias sociales (Coneval, 2011).

\section{Estudios sobre la medición de la pobreza a nivel regional o intraurbano en México: Una revisión}

En México los estudios en los que se hayan reportado trabajos de medición de la pobreza a nivel regional e intraurbano son escasos. Damián (2009) estimó la pobreza para las delegaciones del Distrito Federal agrupadas por estratos de pobreza (alta, media, media-baja y baja). Posteriormente presenta en forma detallada la situación de carencia en las tres principales dimensiones del MMIP (ingreso, tiempo y NBI) y de los distintos componentes del método de NBI, tanto para el D.F. en su conjunto como para las delegaciones agrupadas por estrato. El trabajo se elaboró con base en la sobre muestra de la Enigh, que el Inegi levantó con financiamiento del gobierno local, en 2004, lográndose así tener información representativa a nivel de la entidad.

Limas (2010) construyó el índice Foster, Greer y Thorbecke (FGT) para diagnosticar la pobreza femenina y masculina en Ciudad Juárez, Chihuahua. Para la construcción del índice FGT usó información del Scince 2000 por colonia en donde incluyeron los resultados definitivos del xir Censo General de Población y Vivienda 2000 e información del Consejo Nacional de Población (Conapo) y Comisión Nacional de los Salarios Mínimos (Conasami). En cuanto a la información básica vale señalar que la base original contiene datos de 728 colonias. Las colonias que se localizan en el poniente de la ciudad presentan mayores porcentajes de población en pobreza extrema. Las mujeres sufren con mayor intensidad la pobreza extrema de manera cotidiana ya que son las que se quedan en sus casas y los hombres salen a trabajar fuera de los lugares en donde viven (Inegi, 2000). 
Caloca y Ortiz (2016) realizaron un estudio para la identificación espacial de la pobreza en la delegación Milpa Alta en la Ciudad de México. El primer nivel de análisis fue a escala de AGEB y posteriormente a nivel de manzana urbana. Para llevar a cabo dicha actividad usaron el método denominado Enfoque Sectorial de Necesidades Básicas Insatisfechas (ESNBI) (Boltvinik y Hernández, 1999) solo para la selección de las variables, y en la estimación emplearon la técnica de componentes principales. Las fuentes de información fueron el Censo de Población y Vivienda de 1990 y 2000 (Inegi, 1990; 2000), para lo cual tomaron en cuenta los AGEB en un principio y manzanas en un segundo lugar.

Cortés, Benegas, Fernández y Mora (2007) estimaron la intensidad y profundidad de la pobreza para el estado de Chiapas a nivel municipal, donde aplicaron la metodología desarrollada por el Comité Técnico para la Medición de la Pobreza. Para llevar a cabo dicha actividad fue necesario generar información de la pobreza a escala municipal, pero ante la imposibilidad de levantar una encuesta por los costos y después de considerar que la Enigh solo tiene representatividad a nivel rural-urbano, se decidió usar la muestra de $10 \%$ que se levantó al mismo tiempo que el xir Censo de Población y Vivienda del 2000. Con base en lo anterior se midió la pobreza por municipio y se construyeron las medidas de intensidad y profundidad.

En general, se puede señalar que en México además de haber pocos estudios que hagan la medición de la pobreza a nivel regional o urbano, la mayoría de ellos usan información generada por el Inegi. La excepción es el estudio sobre la medición de la pobreza en el Distrito Federal en donde se solicitó al Inegi ampliar la muestra de la Enigh (Inegi, 2000).

\section{Metodología}

En el caso de Ciudad Juárez, para realizar la medición multidimensional de la pobreza a nivel intraurbano, se aplicó la metodología "oficial" de la pobreza propuesta por Coneval. Para su adecuación a nivel intraurbano se siguieron varios pasos que incluyen desde la selección de las UTA, la selección de la muestra, el levantamiento de la encuesta, la construcción de la base de datos y la aplicación del algoritmo de Coneval para el procesamiento de la base de datos en spss.

\section{Unidades territoriales de análisis}

Uno de los principales retos en la aplicación de la metodología usada por el Coneval para medir la pobreza a nivel de AGEB es la necesidead del levantamiento de una gran cantidad de cuestionarios para que los resultados sean estadísticamente representativos, lo que incrementa los costos del operativo de campo. Por lo anterior, se propuso como alternativa la construcción de las UTA que más adelante detallaremos, así como los criterios metodológicos para su construcción.

Las UTA forman la base del muestreo y están constituidas por agrupaciones de viviendas con características similares dependiendo del estrato al que pertenecen. El tamaño del rango de una UTA fue de 1191 a 9226 viviendas habitadas, y pueden formarse a partir de un AGEB. El criterio para la unión de dos o más AGEB es que pertenezcan al mismo estrato socioeconómico. 


\section{Estratificación}

La estratificación considera las características sociodemográficas de los habitantes de las viviendas de Ciudad Juárez, referidas particularmente al ingreso. ${ }^{5}$ Dichas características se expresan a través de 13 indicadores sociodemográficos construidos mediante métodos estadísticos multivariados, en específico agrupamiento o clustering a partir de la información del XIII Censo de Población y Vivienda 2010 a nivel de AGEB.

\section{Esquema de muestreo}

El levantamiento de la encuesta para Ciudad Juárez, se diseñó bajo un esquema de muestreo probabilístico, estratificado, bietápico y por conglomerados.

\section{Tamaño de la muestra}

Para el cálculo del tamaño de la muestra se consideró como variable de referencia el promedio del ingreso del estado de Chihuahua, obtenido mediante datos de la Enigh 2010. La expresión utilizada fue:

$$
\mathbf{n}=\frac{\mathbf{z}^{2} s^{2} D E F F}{r^{2} \overline{\mathbf{X}}^{2}(\mathbf{1}-t n r) P H V}
$$

Donde:

\begin{tabular}{|c|c|}
\hline $\mathbf{n}$ & Tamaño de muestra \\
\hline $\mathbf{Z}$ & $\begin{array}{l}\text { Valor asentado en las tablas estadísticas de la distribución normal } \\
\text { estándar para alcanzar } 90 \% \text { de confianza prefijada }\end{array}$ \\
\hline$\underline{s}^{2}$ & Estimación de la varianza poblacional de la variable de interés \\
\hline$\overline{\mathbf{X}} \overline{\mathbf{X}}$ & Estimación del promedio de la variable de interés \\
\hline DEFF & $\begin{array}{l}\text { Efecto de diseño definido como el cociente de la } \\
\text { varianza en la estimación del diseño utilizado entre la varianza } \\
\text { obtenida considerando un muestreo aleatorio simple para un mismo } \\
\text { tamaño de muestra }\end{array}$ \\
\hline$r$ & Error relativo máximo aceptable \\
\hline $\operatorname{tnr}$ & Tasa de no respuesta máxima esperada \\
\hline$P H V$ & Promedio de hogares por vivienda \\
\hline
\end{tabular}

Fijando un nivel de confianza de $90 \%$, un efecto de diseño de $3.3^{* *}$, una varianza poblacional de $2032125^{* *}$, un error relativo máximo aceptable de $3.5 \%$, un promedio de ingreso corriente total por hogar de 14896 , una tasa de no respuesta máxima esperada de $15 \%$ y un promedio de hogares por vivienda de 1.02 , se determinó

\footnotetext{
${ }^{5}$ Debido a que no existen estimaciones de ingreso a nivel AGEB, se usaron indicadores "proxy" que ayudaron a formar
} los estratos. 
una muestra de 6887 viviendas. La muestra se proporcionó a $10 \%{ }^{6}$ y se ajustó a 688 viviendas. Una vez definidas las uTA se asignó como mínimo de 5 cuestionarios y quedaron 694 cuestionarios por cubrir.

\section{Afijación de la muestra}

Para asignar la muestra al nivel urbano que comprende el estudio, se consideró un número básico de 688 viviendas, distribuidas aproximadamente igual entre las 65 uta (Véase Figura 1). La población objetivo fue el hogar, por lo cual, al interior de las UTA se le asignó de manera proporcional al número de viviendas del marco obtenido a partir de la cobertura geográfica de AGEB de 2010. Con ello se asegura una cobertura total del área de estudio. Adicionalmente, se consideró un total de 694 viviendas, para complementar el universo de muestra considerado, en caso de una no respuesta de $15 \%$.

\section{Selección de la muestra}

En el apartado se estima la probabilidad de que cada una de las viviendas sea seleccionada para ser encuestada. La selección de la muestra se realiza de manera independiente para cada UTA y el procedimiento deberá adecuarse a un proceso aleatorio para cada una de las zonas mencionadas, mediante el siguiente método general:

1. De las $n_{t}$ UTA que integran el marco de la muestra maestra, se seleccionarán todas las UTA con igual probabilidad.

2. En cada UTA se seleccionaron $X_{n}$ viviendas con igual probabilidad para la muestra estimada.

Por lo tanto, la probabilidad de seleccionar una vivienda de la i-ésima UTA, del h-ésimo estrato de Ciudad Juárez es:

$$
P\left\{V_{i}\right\}=\frac{n_{i} m_{i}}{N_{i} M_{i}}
$$

Donde:

ni número de viviendas seleccionadas, en la i-esima UTA

mi número de vivienda seleccionadas, en el h-esimo estrato

$\mathrm{Ni}$ número de viviendas, en la i-esima UTA

Mi Mi número de viviendas, en el h-esimo estrato

\footnotetext{
${ }^{6}$ La justificación del tamaño de la muestra se fundamenta en el hecho de que la selección de 10\% permite que el error se mantenga dentro de un error aceptable y el beneficio de incrementar la muestra a un error menor es muy marginal.
} 
Figura 1: Formación de unidades territoriales de análisis en Ciudad Juárez (2010)

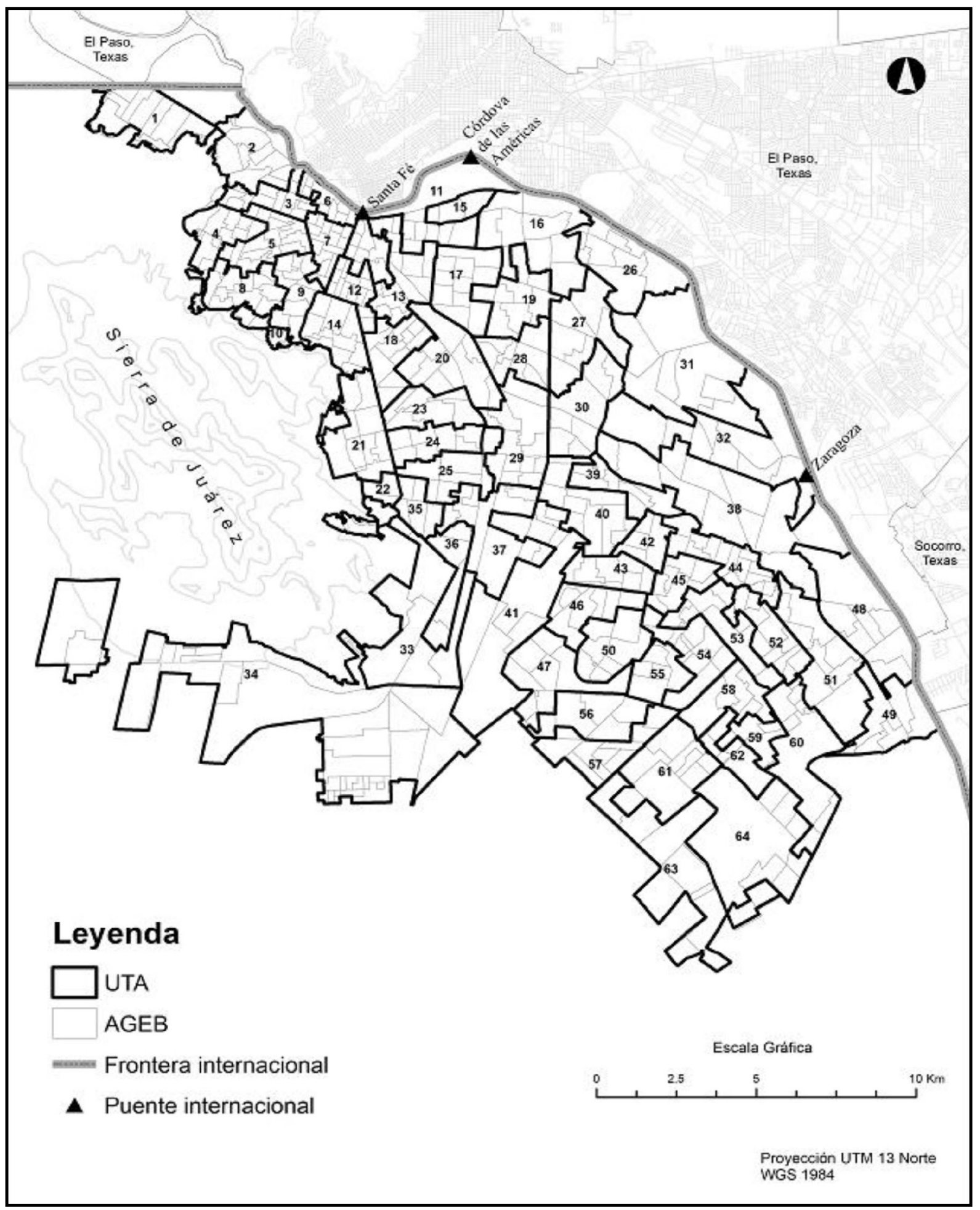

Fuente: Elaboración propia con base en el XIII Censo de Población y Vivienda (Inegi, 2010). 


\section{Cuestionario}

Una vez que se construyeron las UTA se diseñó el cuestionario, que para los propósitos de este estudio se incluyeron los mismos reactivos que el MCs de la Enigh que aplica el Inegi. Lo anterior, con el objetivo de hacer comparativos los resultados con la medición global de la pobreza realizada por Coneval para el municipio de Juárez. El levantamiento de la encuesta se llevó a cabo entre los meses de noviembre de 2011 a febrero de 2012.

\section{Construcción de la base de datos}

En los meses de marzo a mayo se capturó la información y se hicieron los primeros cuadros para identificar posibles inconsistencias de la captura y hacer la limpieza de la base de datos.

\section{Aplicación de algoritmo}

Para la medición de la pobreza a nivel intraurbano se utilizó el algoritmo desarrollado por Coneval para la medición de la pobreza multidimensional. La información fue procesada en el programa sPss (Statistics 20).

\section{El contexto de la pobreza en Ciudad Juárez, Chihuahua y su medición a nivel municipal}

Diversos estudios señalaron que los estados de la frontera norte fueron los que tuvieron mayor éxito en reducir la pobreza entre 1970 y el año 2000 (Anderson, 2003). Además, que estos mismos estados presentaron un menor porcentaje de su población en pobreza que el promedio nacional, sobre todo pobreza extrema (Camberos y Bracamontes, 1995). De la misma manera, Anderson y Gerber (2009) mostraron que la diferencia del ingreso per cápita entre los condados fronterizos del sur de Estados Unidos y los municipios fronterizos de México era menor si se les comparaba con otras regiones de México. Lo anterior, era explicado por el rápido crecimiento económico de la región, que se caracteriza por una alta generación de empleos en la industria maquiladora, lo que permitía que los grupos sociales usaran estrategias de sobrevivencia como la basada en compartir varios y distintos tipos de ingreso entre los miembros del hogar. A este modelo de sobrevivencia se le denominó "los recursos de la pobreza" (González, 1986), el cual depende de la existencia y disponibilidad de una gran cantidad de empleos en el mercado local.

En el caso del estado de Chihuahua, y en específico del municipio de Juárez, el panorama anterior se modificó de manera radical a partir del año 2000 como resultado de dos eventos: la pérdida en la dinámica del empleo manufacturero como resultado de la recesión de la economía de Estados Unidos y una profunda crisis de inseguridad que dejó miles de personas sin ingresos. Por un lado, la economía local a partir del año 2000 no solo redujo su tasa de crecimiento del empleo manufacturero, sino que se perdieron 
más de 80000 empleos (Fuentes, 2009). Para el año 2005, la economía local creció, pero no alcanzó el número de empleos que se tenían en el año 2000 y a partir del año 2007 mostró nuevamente una caída del empleo producto de la recesión de la economía global (Fuentes y Peña, 2010).

Por otro lado, la crisis de inseguridad surgió como resultado del papel de la ciudad como espacio estratégico de las economías ilegales, en específico del flujo de drogas prohibidas hacia el mercado de Estados Unidos (Fuentes y Peña, 2017). En ese contexto, se dio el enfrentamiento entre cárteles rivales por el control de las rutas del tráfico de drogas y los mercados locales que dejó miles de personas muertas y desaparecidas. Dicha situación tuvo dos efectos, por una parte, el cierre de cientos de comercios y servicios ante el incremento de delitos como el secuestro y extorsiones a negocios que condujo a que miles de personas se quedaron sin empleos. Por otra parte, entre los costos sociales de la violencia se contabilizan 9262 homicidios entre 2008 y 2012 (Monárrez y Cervera, 2013), la mayoría de ellos hombres jóvenes. Lo anterior, ocasionó que miles de hogares perdieran a sus proveedores económicos principales y que cientos de viudas, huérfanos, padres y hermanos se quedaran en el desamparo. La combinación de los dos factores antes mencionados exacerbó la pobreza en la localidad a partir del 2007.

De igual manera, la pérdida del empleo no solo significa quedarse sin ingresos sino que también implica quedarse sin derechos sociales como derechohabiencia a servicios de salud, seguridad social, educación y vivienda. Lo que se traduce en que por periodos muy cortos de tiempo la población pueda mostrar carencias y, de acuerdo con la metodología de la medición multidimensional de la pobreza, el grueso de la población rápidamente transite de ser pobres vulnerables a pobres moderados o extremos en algunos casos.

En este contexto, de acuerdo con las mediciones de Coneval (2010) en el periodo de 2008 a 2010 el estado de Chihuahua fue uno de los que tuvo un mayor aumento en el número de personas en situación de pobreza en el país. Lo anterior, debido a que sus dos principales áreas urbanas; Ciudad Juárez y Chihuahua, experimentaron con mayor agudeza los impactos locales de la recesión de la economía global y de la economía ilícita. En este contexto, la Dirección de Desarrollo Social del gobierno municipal de Juárez se vio superada por el incremento en las solicitudes de apoyos económicos o en especie de cada uno de sus programas sociales, sobre todo en el que se refiere a despensas familiares. Por lo anterior, decidió solicitar la medición de la pobreza pero ahora a nivel intraurbano con los siguientes objetivos: 1) tener una nueva medición de la pobreza, 2) negociar con otros niveles de gobierno mayores recursos y 3) focalizar sus programas sociales.

Los resultados de la medición muestran que en el año 2012 el porcentaje de personas en pobreza representó $39.8 \%$, el cual es mayor a la estimada por Coneval para el estado de Chihuahua (35.3\%). Por su parte, el porcentaje de personas en pobreza extrema representó $5.6 \%$, lo que significa $1.8 \%$ más que la pobreza estimada para el estado de Chihuahua (3.8\%). La población vulnerable con carencias sociales y por ingresos alcanzó $22 \%$ y $15.9 \%$ respectivamente. Finalmente, la población no pobre multidimensional y no vulnerable alcanzó $22.3 \%$ (Véase Tabla 1).

En lo que respecta a la privación social $64.3 \%$ de la población presenta al menos una carencia social y $11.0 \%$ con al menos tres carencias sociales. Además, $31.6 \%$ (377 314 personas) de la población muestran carencias por rezago de acceso a seguridad social; 31.4\% (786 008 personas) presentan carencias por rezago de 
acceso a servicios de salud; $26.3 \%$ (317 179 personas) exhiben carencias por rezago educativo; 29.9\% (455 605 personas) exponen carencias por rezago de calidad y espacios de la vivienda; $23.6 \%$ (355 605 personas) presenta carencias por rezago de acceso a la alimentación.

Tabla 1: Indicadores de la medición de la pobreza en el municipio de Juárez, Chihuahua, 2012

\begin{tabular}{lr} 
Indicadores de incidencia & $\%$ \\
\hline Pobreza & \\
Población en situación de pobreza & 39.8 \\
Población en situación de pobreza moderada & 34.2 \\
Población en situación de pobreza extrema & 5.6 \\
Población vulnerable por carencias sociales & 22.0 \\
Población vulnerable por ingresos & 15.9 \\
Población no pobre multidimensional y no vulnerable & 22.3 \\
Privación social & \\
Población con al menos una carencia social & 64.3 \\
Población con al menos tres carencias sociales & 11.0 \\
Indicadores de carencia social & \\
Rezago educativo & 26.3 \\
Carencia por acceso a los servicios de salud & 31.4 \\
Carencia por acceso a la seguridad social & 31.6 \\
Carencia por calidad de espacios de la vivienda & 29.9 \\
Carencia por acceso a los servicios básicos de la vivienda & 12.7 \\
Carencia por acceso a la alimentación & 23.6 \\
Bienestar & \\
\hline Población con un ingreso inferior a la línea de bienestar mínimo & 15.0 \\
\hline
\end{tabular}

Fuente: Fuentes, Peña y Hernández (2012).

Por lo que toca al ingreso, $56 \%$ de la población tiene un ingreso inferior a la línea del bienestar y $15 \%$ tiene un ingreso inferior a la línea de bienestar mínimo. Lo cual muestra que de tener carencias sociales un alto porcentaje de la población se podría clasificar como pobres moderados o extremos.

\section{Medición de la pobreza multidimensional a nivel intraurbano en Ciudad Juárez, Chihuahua, 2012}

La distribución espacial de los distintos tipos de pobreza muestra que las UTA que presentan mayor población en situación de pobreza se localizan en el poniente y suroriente de la ciudad. Lo anterior, se debe a que exhiben el mayor número 
Figura 2: Localización de población en condición de pobreza en Ciudad Juárez por UTA (2012)

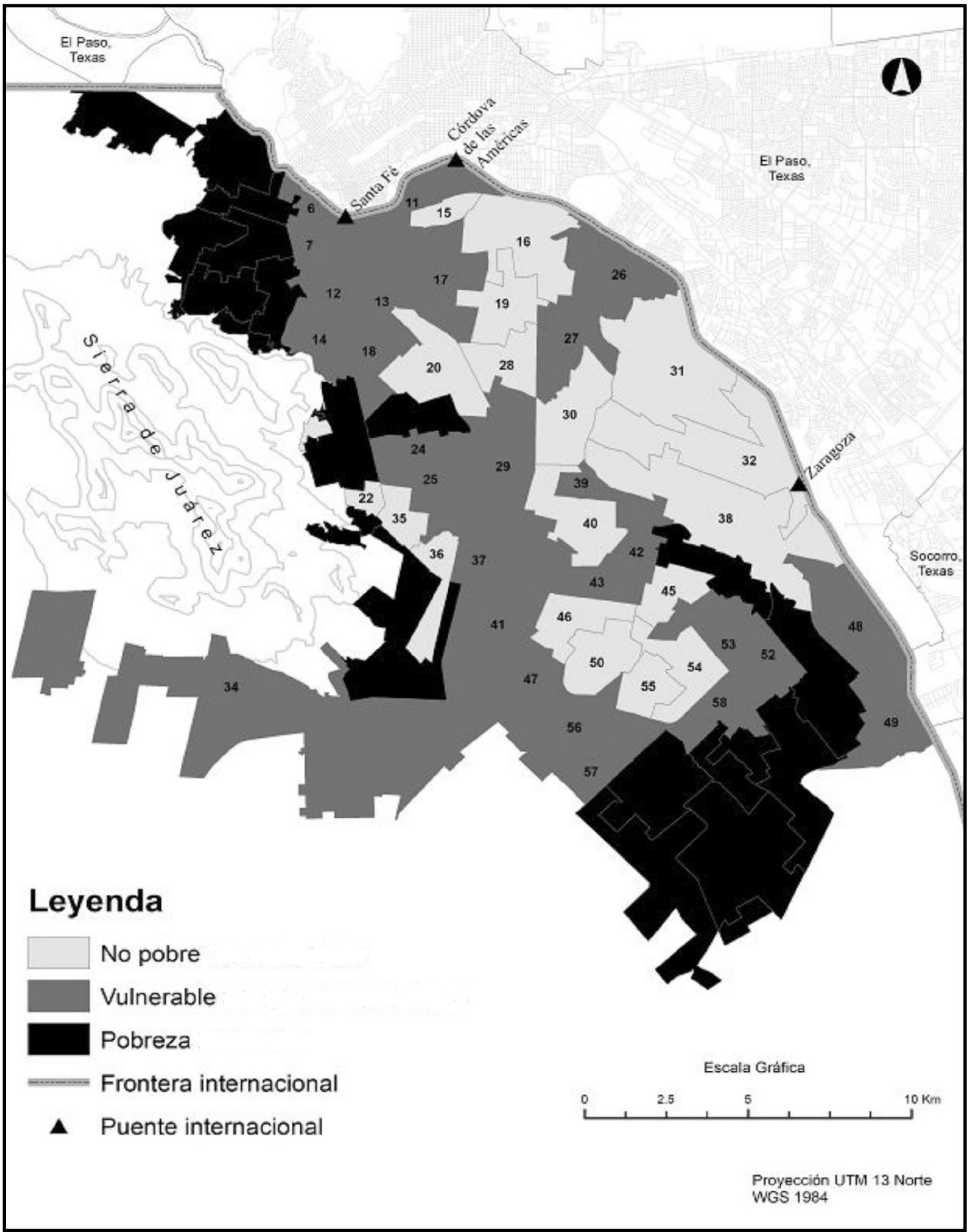

Fuente: Fuentes et al. (2012). 
de carencias promedio con 2.5. ${ }^{7}$ Así mismo, desde el centro histórico hacia el sur existe un agrupamiento de UTA que es vulnerable por carencias sociales. Finalmente, en el nororiente se ubican los UTA que concentran población que no es pobre (Véase Figura 2).

En la ciudad existen tres conglomerados de pobreza extrema. El primero se localiza en el extremo norponiente en la zona conocida como Puerto Anapra, la cual se fundó mediante mecanismos de invasión de terrenos por lo que se presenta una baja cobertura de servicios públicos. En esa zona se localiza la UTA 2 en la que 50\% de la población se encuentra en pobreza extrema, algo similar sucede con la UTA 3 en la cual $42 \%$ de su población está en pobreza extrema. El segundo agrupamiento de UTA en donde existe población en situación de pobreza extrema se ubica en el surponiente sobre la salida a la carretera que conecta a Ciudad Juárez con el estado de Sonora. Dichos asentamientos son zonas de crecimiento informal caracterizado por grandes rezagos de infraestructura. En esa zona $38 \%$ de la población se encuentra en pobreza extrema y el restante $62 \%$ en pobreza moderada. El tercer conglomerado se localiza en el suroriente en zonas de crecimiento formal a través de programas de vivienda económica. En esa área se ubica la uTA 34 en donde $38 \%$ de la población se encuentra en situación de pobreza extrema (Véase Tabla 2b).

En contraste, las zonas de la ciudad en donde se agrupan principalmente población no pobre se localizan en el nororiente o cercanas a las principales vialidades que comunican al suroriente de la ciudad. Por ejemplo, en la UTA número $15,70 \%$ de su población no es pobre (Tabla 2a), lo mismo sucede con la UTA 46 y 50 en los cuales $66 \%$ y $61 \%$ de la población no es pobre respectivamente (Véase Tabla $2 \mathrm{~b}$ ).

La falta de acceso a la seguridad social es el rubro que más contribuye en esta medida de pobreza (Véase Tabla 3a). La carencia por rezago en seguridad social se refiere a las personas que no tienen acceso a beneficios como pensiones o jubilaciones. La carencia para toda la localidad alcanzó $31.6 \%$ de la población y en términos de su distribución espacial las UTA que tienen los mayores rezagos se agrupan en el norponiente de la ciudad. Por ejemplo, en la uTAS número 2 y $3,59 \%$ y $38.7 \%$ de la población exhibe dicho rezago. De igual manera, existe otro conglomerado de uTA que se localizan en las colonias que rodean al Centro Histórico como las uTA 10 y 11 en las que 53.3\% y $61.5 \%$ de los residentes muestran dichas carencias. Entre las colonias que tienen altos porcentajes de la población en esa situación se encuentran San Borja, Franja del Río, Sara Lugo, Ladrillera Juárez, Bosques del Valle, Cuauhtémoc, etc.

La carencia por servicios de salud permaneció en segundo lugar y los agrupamientos de este rezago se ubican en el norponiente y en las colonias que rodean al Centro Histórico. Del norponiente, por ejemplo, en las UTA número 2 y $3,55.6 \%$ y $32 \%$ de la población no cuenta con derechohabiencia a servicios de salud respectivamente. Sin embargo, la concentración de personas con carencia por servicios de salud se localiza en las colonias que rodean al Centro Histórico, en donde principalmente viven personas de la tercera edad que se han quedado sin servicios de salud. Por ejemplo, las UTA 11, 12 y 13 tienen $46 \%, 46 \%$ y $51 \%$ de su población con rezagos en servicios de salud respectivamente (Tabla 3a). Para propósitos de focalización de políticas públicas relacionadas con la salud pública se pueden intervenir colonias como Franja del Río, Ampliación Ladrillera Juárez, Sara Lugo, Ladrillera Juárez, Oscar González, Buendía, Cumbres, Paseo del Parque, etc.

\footnotetext{
${ }^{7}$ La medición de la pobreza multidimensional para hacer la medición usa dos criterios uno es el ingreso por debajo de la línea de bienestar y el incumplimiento de algunos de los derechos a la salud, educación, seguridad social, etc., (carencias). Entonces, es posible determinar el número promedio de carencias que tiene cada UTA.
} 
La carencia por calidad y espacios de la vivienda se ubicó en tercer lugar como fuente de pobreza. Dicha carencia se concentra en la periferia urbana sobre todo en el poniente. Por ejemplo, en la UTA $64,82 \%$ de las personas que viven en dicho lugar presentan la carencia. Por lo anterior, es importante focalizar territorialmente

Tabla 2a: Porcentaje de la población en pobreza moderada, pobreza extrema, no pobre, carencia de acceso a servicios de salud y educativos por UTA (2012)

\begin{tabular}{|c|c|c|c|}
\hline UTA & Pobreza extrema & Pobreza moderada & No pobre \\
\hline 1 & 34.78 & 65.22 & 0.00 \\
\hline 2 & 50.00 & 50.00 & 0.00 \\
\hline 3 & 41.94 & 48.39 & 9.68 \\
\hline 4 & 7.14 & 75.00 & 17.86 \\
\hline 5 & 50.00 & 42.86 & 7.14 \\
\hline 6 & 0.00 & 81.25 & 18.75 \\
\hline 7 & 25.00 & 58.34 & 16.67 \\
\hline 8 & 30.30 & 45.46 & 24.24 \\
\hline 9 & 25.71 & 42.86 & 31.43 \\
\hline 10 & 0.00 & 80.00 & 20.00 \\
\hline 11 & 38.46 & 50.00 & 11.54 \\
\hline 12 & 4.88 & 68.29 & 26.83 \\
\hline 13 & 25.00 & 36.11 & 38.89 \\
\hline 14 & 4.08 & 81.63 & 14.29 \\
\hline 15 & 7.69 & 23.08 & 69.23 \\
\hline 16 & 0.00 & 76.67 & 23.33 \\
\hline 17 & 32.43 & 43.24 & 24.33 \\
\hline 18 & 19.05 & 66.67 & 14.29 \\
\hline 19 & 3.45 & 37.93 & 58.62 \\
\hline 20 & 2.94 & 44.12 & 52.94 \\
\hline 21 & 27.27 & 45.45 & 27.27 \\
\hline 22 & 0.00 & 75.00 & 25.00 \\
\hline 23 & 11.54 & 76.92 & 11.54 \\
\hline 24 & 28.94 & 63.16 & 7.89 \\
\hline 25 & 16.32 & 46.94 & 36.74 \\
\hline 26 & 40.00 & 0.00 & 60.00 \\
\hline 27 & 0.00 & 73.08 & 26.92 \\
\hline 28 & 9.37 & 31.25 & 59.38 \\
\hline 29 & 24.39 & 53.66 & 21.95 \\
\hline 30 & 0.00 & 30.77 & 69.23 \\
\hline 31 & 0.00 & 33.34 & 66.66 \\
\hline 32 & 6.06 & 57.58 & 36.36 \\
\hline
\end{tabular}

Fuente: Fuentes et al. (2012). 
programas de mejoramiento de la vivienda en colonias como Municipio libre, Periodista, Telegrafistas, Rancho Anapra, Frida Khalo, Fray García de San Francisco, etc. (Véase Tabla 3b).

Tabla 2b: Porcentaje de la población en pobreza moderada, pobreza extrema, no pobres, carencia de acceso a servicios de salud y educativos por UTA (2012)

\begin{tabular}{|c|c|c|c|}
\hline UTA & Pobreza extrema & Pobreza moderada & No pobre \\
\hline 33 & 11.11 & 77.78 & 11.11 \\
\hline 34 & 38.46 & 42.31 & 19.23 \\
\hline 35 & 0.00 & 57.14 & 42.86 \\
\hline 36 & 0.00 & 54.76 & 45.24 \\
\hline 37 & 16.00 & 76.00 & 8.00 \\
\hline 38 & 6.25 & 50.00 & 43.75 \\
\hline 39 & 23.08 & 46.15 & 30.77 \\
\hline 40 & 9.52 & 45.24 & 45.24 \\
\hline 41 & 0.00 & 81.25 & 18.75 \\
\hline 42 & 16.13 & 61.29 & 22.58 \\
\hline 43 & 13.89 & 47.22 & 38.89 \\
\hline 44 & 18.92 & 45.95 & 35.14 \\
\hline 45 & 0.00 & 69.45 & 30.55 \\
\hline 46 & 0.00 & 33.93 & 66.07 \\
\hline 47 & 7.69 & 69.23 & 23.08 \\
\hline 48 & 16.67 & 77.08 & 6.25 \\
\hline 49 & 40.00 & 48.00 & 12.00 \\
\hline 50 & 4.11 & 34.25 & 61.64 \\
\hline 51 & 11.11 & 68.52 & 20.37 \\
\hline 52 & 8.57 & 60.00 & 31.43 \\
\hline 53 & 19.36 & 38.71 & 41.93 \\
\hline 54 & 2.04 & 36.74 & 61.22 \\
\hline 55 & 2.86 & 37.14 & 60.00 \\
\hline 56 & 9.52 & 61.90 & 28.57 \\
\hline 57 & 28.95 & 39.47 & 31.58 \\
\hline 58 & 26.47 & 50.00 & 23.53 \\
\hline 59 & 5.71 & 68.57 & 25.71 \\
\hline 60 & 12.20 & 60.98 & 26.83 \\
\hline 61 & 8.77 & 63.16 & 28.07 \\
\hline 62 & 23.08 & 65.38 & 11.54 \\
\hline 63 & 8.11 & 45.95 & 45.95 \\
\hline 64 & 9.09 & 63.64 & 27.27 \\
\hline
\end{tabular}

Fuente: Fuentes et al. (2012). 
Tabla 3a: Porcentaje de carencia social en Ciudad Juárez, Chihuahua, por UTA (2012)

\begin{tabular}{|c|c|c|c|c|}
\hline UTA & $\begin{array}{l}\text { Carencia por } \\
\text { servicios de } \\
\text { salud }\end{array}$ & $\begin{array}{l}\text { Carencia por } \\
\text { servicios } \\
\text { educativos }\end{array}$ & $\begin{array}{l}\text { Carencia por } \\
\text { rezago en } \\
\text { seguridad social }\end{array}$ & $\begin{array}{c}\text { Carencia por calidad } \\
\text { y espacio de la } \\
\text { vivienda }\end{array}$ \\
\hline 1 & 28.6 & 31.8 & 26.1 & 51.4 \\
\hline 2 & 55.6 & 21.9 & 59.4 & 17.1 \\
\hline 3 & 32.0 & 46.9 & 38.7 & 40.8 \\
\hline 4 & 10.7 & 39.3 & 10.7 & 10.3 \\
\hline 5 & 33.3 & 57.1 & 35.7 & 44.7 \\
\hline 6 & 33.3 & 31.2 & 43.8 & 0.0 \\
\hline 7 & 38.1 & 20.8 & 29.2 & 25.0 \\
\hline 8 & 29.4 & 25.8 & 39.4 & 22.9 \\
\hline 9 & 38.7 & 28.6 & 42.9 & 22.5 \\
\hline 10 & 25.0 & 33.3 & 53.3 & 33.3 \\
\hline 11 & 45.5 & 34.6 & 61.5 & 13.8 \\
\hline 12 & 46.2 & 36.6 & 24.4 & 0.0 \\
\hline 13 & 51.4 & 13.9 & 47.2 & 0.0 \\
\hline 14 & 26.5 & 29.2 & 36.7 & 0.0 \\
\hline 15 & 7.7 & 7.7 & 7.7 & 91.7 \\
\hline 16 & 26.9 & 10.0 & 30.0 & 0.0 \\
\hline 17 & 45.5 & 24.3 & 51.4 & 81.7 \\
\hline 18 & 43.2 & 38.1 & 50.0 & 0.0 \\
\hline 19 & 32.1 & 13.8 & 34.5 & 76.2 \\
\hline 20 & 25.0 & 38.2 & 26.5 & 75.0 \\
\hline 21 & 31.0 & 42.4 & 39.4 & 23.7 \\
\hline 22 & 40.0 & 0.0 & 35.0 & 24.0 \\
\hline 23 & 20.8 & 34.6 & 23.1 & 0.0 \\
\hline 24 & 30.6 & 63.2 & 42.1 & 0.0 \\
\hline 25 & 30.4 & 32.7 & 22.4 & 0.0 \\
\hline 26 & 40.0 & 0.0 & 40.0 & 0.0 \\
\hline 27 & 48.0 & 11.5 & 53.8 & 0.0 \\
\hline 28 & 31.3 & 15.6 & 31.3 & 0.0 \\
\hline 29 & 52.6 & 26.8 & 46.3 & 15.6 \\
\hline 30 & 19.2 & 0.0 & 19.2 & 0.0 \\
\hline 31 & 22.2 & 0.0 & 33.3 & 0.0 \\
\hline 32 & 35.5 & 24.2 & 36.4 & 0.0 \\
\hline
\end{tabular}

Fuente: Fuentes et al. (2012). 
Tabla 3b: Porcentaje de carencia social en Ciudad Juárez, Chihuahua, por UTA (2012)

\begin{tabular}{|c|c|c|c|c|}
\hline UTA & $\begin{array}{l}\text { Carencia por } \\
\text { servicios de salud }\end{array}$ & $\begin{array}{c}\text { Carencia por } \\
\text { servicios educativos }\end{array}$ & $\begin{array}{l}\text { Carencia por } \\
\text { rezago en } \\
\text { seguridad social }\end{array}$ & $\begin{array}{l}\text { Carencia por } \\
\text { calidad y espacio } \\
\text { de la vivienda }\end{array}$ \\
\hline 33 & 28.0 & 25.9 & 29.6 & 78.0 \\
\hline 34 & 42.3 & 34.6 & 46.2 & 40.7 \\
\hline 35 & 9.4 & 37.1 & 14.3 & 0.0 \\
\hline 36 & 21.9 & 16.7 & 19.0 & 14.3 \\
\hline 37 & 47.9 & 16.0 & 44.0 & 8.0 \\
\hline 38 & 34.4 & 21.9 & 31.3 & 0.0 \\
\hline 39 & 30.8 & 25.6 & 28.2 & 10.7 \\
\hline 40 & 41.5 & 45.2 & 40.5 & 9.8 \\
\hline 41 & 18.8 & 21.9 & 15.6 & 0.0 \\
\hline 42 & 41.9 & 35.5 & 35.5 & 0.0 \\
\hline 43 & 38.9 & 11.1 & 27.8 & 13.3 \\
\hline 44 & 40.5 & 21.6 & 43.2 & 18.6 \\
\hline 45 & 22.2 & 16.7 & 25.0 & 0.0 \\
\hline 46 & 12.5 & 25.0 & 14.3 & 0.0 \\
\hline 47 & 19.2 & 53.8 & 7.7 & 15.2 \\
\hline 48 & 39.6 & 14.6 & 33.3 & 37.5 \\
\hline 49 & 41.7 & 28.0 & 32.0 & 41.9 \\
\hline 50 & 11.8 & 16.4 & 15.1 & 0.0 \\
\hline 51 & 40.7 & 14.8 & 38.9 & 20.0 \\
\hline 52 & 28.6 & 17.1 & 28.6 & 32.6 \\
\hline 53 & 29.5 & 19.4 & 29.0 & 22.4 \\
\hline 54 & 25.0 & 20.4 & 22.4 & 0.0 \\
\hline 55 & 20.0 & 22.9 & 11.4 & 0.0 \\
\hline 56 & 29.3 & 19.0 & 21.4 & 16.3 \\
\hline 57 & 18.4 & 23.7 & 26.3 & 29.0 \\
\hline 58 & 25.0 & 41.2 & 29.4 & 37.8 \\
\hline 59 & 22.9 & 20.0 & 17.1 & 17.9 \\
\hline 60 & 41.5 & 29.3 & 26.8 & 12.8 \\
\hline 61 & 36.8 & 36.8 & 29.8 & 0.0 \\
\hline 62 & 38.5 & 46.2 & 23.1 & 40.0 \\
\hline 63 & 8.3 & 35.1 & 16.2 & 13.6 \\
\hline 64 & 23.3 & 36.4 & 27.3 & 82.0 \\
\hline
\end{tabular}

Fuente: Fuentes et al. (2012). 
El cálculo de la carencia por rezago educativo para toda la ciudad alcanza a $26.3 \%$ de la población y se coloca en el cuarto lugar como fuente de la pobreza. Los agrupamientos de UTA con rezago educativo se localizan en el norponiente y suroriente. En el norponiente se concentran los mayores rezagos tales como en las UTA número $3,4,5,14,18$ y 24, en las que el porcentaje de población con esta carencia oscila de $26.5 \%$ a $63 \%$. De igual manera, en el suroriente hay un conglomerado de menor tamaño pero en donde existen UTA que tienen más de $50 \%$ de sus residentes con dicho rezago social. Las principales colonias que tienen un mayor atraso educativo son la Periodista, San Felipe del Real, Torreón, Luis Olague, Constituyentes, Santa Lucia, Valle de los Cantaros, Valle de los Olivos, Puente del Bravo, entre otras.

Las dos carencias que registraron los porcentajes de población más bajos fueron; la carencia por acceso a la alimentación y la carencia por acceso a los servicios básicos de la vivienda.

En el espacio económico, la población con ingreso inferior a la línea de bienestar mínimo (LBM) representó 33.6\% de la población y la mayor parte de ella se concentra en el norponiente y suroriente. Los UTA 2, 7 y 8 tienen más de $60 \%$ de su población con dicha problemática, las colonias con población con mayores rezagos son San Borja, Miguel Auza, Franja del Río, El Sauzal, Francisco Villa, Lomas del Valle, La Mesita, Ladrillera Juárez, Sara Lugo, etc.

Para continuar con la medición de la pobreza multidimensional es necesario contar con una serie de medidas que indiquen la magnitud y estado de la pobreza de la población tales como la incidencia, intensidad y profundidad. "Las medidas de profundidad $^{8}$ e intensidad ${ }^{9}$ de la pobreza son valiosas para conocer qué tan pobres son las personas tanto en el aspecto económico como en el de las carencias sociales" (Coneval, 2017, p. 44).

En la Tabla 4 se presentan las mediciones de la intensidad de la pobreza por UTA en Ciudad Juárez. La intensidad de la pobreza es mayor en las UTA del norponiente tales como la 1, 2, 3 y en las del suroriente como las uta 44, 61, 63 y 64 (Véase la Tabla 4). Además, en las UTA número 1,2 y 3 no solo hay una proporción mayor de pobres sino que también la profundidad de la pobreza es más elevada al alcanzar al .37, .38 y .35. Lo anterior, es debido a que en promedio presentan un mayor número de carencias $(2.25,2.32$ y 2.39). En contraste, en las uTA número 61, 63 y 64 los habitantes tienenuna menor profundidad de la pobreza: .18, .14, y .17 respectivamente; ello es explicado por tener en promedio el menor número de carencias, $1.07, .80$ y 1.05 .

Por su parte, la incidencia muestra que las UTA 1 y 2 tienen un valor de 1.0 lo que significa que $100 \%$ de sus residentes se encuentran en situación de pobreza. Sin embargo, la intensidad en la UTA 2 es menor que en la UTA 1 por lo que en la última la población es menos pobre.

\footnotetext{
${ }^{8}$ La medida de profundidad en el espacio del bienestar económico permite conocer la brecha o distancia promedio del ingreso de las personas con un ingreso menor de la LB respecto de esta misma línea. En el espacio de los derechos sociales, la medida de profundidad ayuda a identificar el número promedio y la proporción promedio de carencias sociales que padece un grupo de población e identificar cuáles son las privaciones que habrán de superarse (Coneval, 2017).

${ }^{9}$ La medida de intensidad cuantifica el número total de carencias de los conjuntos de población en situación de pobreza, pobreza extrema y moderada en relación con el número máximo total de carencias que puede experimentar la población (Coneval, 2017).
} 
Tabla 4: Intensidad, profundidad e incidencia de la pobreza multidimensional por UTA en Ciudad Juárez, Chihuahua (2012)

\begin{tabular}{|c|c|c|c|}
\hline UTA & Incidencia & Profundidad & Intensidad \\
\hline 2 & 1.00 & 0.38 & 0.38 \\
\hline 5 & 0.93 & 0.40 & 0.37 \\
\hline 1 & 1.00 & 0.37 & 0.37 \\
\hline 3 & 0.90 & 0.35 & 0.32 \\
\hline 62 & 0.88 & 0.31 & 0.28 \\
\hline 8 & 0.76 & 0.30 & 0.22 \\
\hline 23 & 0.88 & 0.24 & 0.22 \\
\hline 33 & 0.89 & 0.22 & 0.20 \\
\hline 51 & 0.80 & 0.23 & 0.18 \\
\hline 21 & 0.73 & 0.24 & 0.18 \\
\hline 4 & 0.82 & 0.21 & 0.17 \\
\hline 9 & 0.69 & 0.24 & 0.17 \\
\hline 10 & 0.80 & 0.20 & 0.16 \\
\hline 60 & 0.73 & 0.22 & 0.16 \\
\hline 44 & 0.65 & 0.20 & 0.13 \\
\hline 59 & 0.74 & 0.18 & 0.13 \\
\hline 61 & 0.72 & 0.18 & 0.13 \\
\hline 64 & 0.73 & 0.17 & 0.13 \\
\hline 63 & 0.54 & 0.14 & 0.08 \\
\hline
\end{tabular}

Fuente: Fuentes et al. (2012).

\section{Conclusiones}

Los avances llevados a cabo en México en cumplimiento de la ley de desarrollo social han permitido que la sociedad mexicana pueda contar con la medición de la pobreza multidimensional a nivel nacional, estatal y municipal; en los dos primeros casos con una periodicidad de cada dos años y en el tercer caso cada cinco años. Lo anterior, ha permitido que se pueda diseñar una serie de políticas públicas que contribuya a reducir las carencias sociales que la población posee. Sin embargo, no se había podido avanzar en la realización de la medición a nivel intraurbano debido a la representatividad estadística de la fuente de información, lo cual dificulta la focalización territorial de los programas sociales a escala urbana.

En este contexto, el gobierno local de Ciudad Juárez hizo el esfuerzo de medir la pobreza usando la misma metodología de Coneval para generar información confiable a nivel intraurbano, con el propósito de focalizar los programas sociales, 
evaluarlos y replicarlos en el tiempo. Además, usar esta información como un instrumento de negociación de recursos ante instancias de gobierno estatales y federales relacionadas con los temas de educación, salud, vivienda, etc., y así puedan aplicar sus programas en las zonas con mayores carencias sociales.

Lo anterior, es relevante en casos de una profunda crisis de inseguridad como la que experimentó Ciudad Juárez y que dejó a miles de personas sin un proveedor al ser asesinados sus hijos, padres, esposos y hermanos, quedando familias sin sustento económico y derechos sociales. Así mismo, producto de la recesión económica mundial y de la situación de inseguridad se perdieron miles de empleos y con ello el ingreso, la derechohabiencia a servicios médicos, entre otros, de modo que ambos factores contribuyeron al incremento de los indicadores de pobreza en la ciudad.

De manera adicional el gobierno municipal de Juárez buscaba justificar el incremento de los recursos públicos para atender la crisis social por lo que decidió hacer una nueva medición de la pobreza mediante la aplicación de la metodología "oficial"; debido a que en México el resultado de la medición "oficial" de la pobreza se constituye como uno de los criterios para la designación de los recursos del gobierno federal para programas sociales.

La metodología a nivel intraurbano permite identificar a las personas en pobreza en cada una de las UTA y la carencia social que más contribuye a la pobreza con lo cual es factible instrumentar acciones de política pública diferenciadas en cada uno de los casos. De igual manera, las medidas de intensidad, profundidad e incidencia permiten evaluar tanto la dimensión económica como de carencias sociales con el objetivo de focalizar en el territorio las acciones de política pública.

Finalmente, es importante reconocer los retos y limitaciones de realizar la medición a nivel intraurbano en Ciudad Juárez. El principal reto es la falta de recursos para poder realizar un levantamiento de miles de cuestionarios con el objetivo de mejorar la calidad de la estimación. Además de la dificultad para captar información confiable en la encuesta sobre el ingreso en un contexto de extrema violencia en el que las personas no están dispuestas a proporcionar dicha información por razones de seguridad.

\section{Referencias}

Anderson, J. (2003). The us-Mexico Border: A Half Century of Change. The Social Science Journal, 40(4), 535-554.

Anderson, J. y Gerber, J. (2009). Fifty Years of Change on the us-Mexico Border: Growth, Development, and Quality of Life. Estados Unidos de América: University of Texas Press.

Banco Mundial. (1990). The World Development Report 1990: Poverty. Nueva York, Estados Unidos de América: Oxford University Press.

Barneche, P., Bugallo, A., Ferrea, H., Ilarregui, M., Monterde, C., Pérez, V., Santa María, T., S. Serrano, y Angeletti, K. (2010). Métodos de medición de la pobreza: conceptos y aplicaciones en América Latina. Entrelíneas de la Política Económica, 26(4), 31-41.

Beccaria, L. y Minujin, A. (1987). Métodos alternativos para medir la evolución del tamaño de la pobreza (Documento de trabajo, núm. 6). Buenos Aires, Argentina: Documentos del Instituto Nacional de Estadística y Censos (INDEC). 
Boltvinik, J. (1990a). Hacia una estrategia para la superación de la pobreza. En J. Boltvinik, Necesidades Básicas y Desarrollo (pp. 25-50). La Paz, Bolivia: ILPES, ILdis, Instituto de Estudios Sociales de La Haya.

Boltvinik, J. (1990b). Pobreza y necesidades básicas. Conceptos y métodos de medición. Caracas, Venezuela: PNUD.

Boltvinik, J. (1992). El método de medición integrada de la pobreza. Una propuesta de su desarrollo. Comercio Exterior, 42(4), 354-365.

Boltvinik, J. (2003). Tipología de los métodos de medición de la pobreza. Los métodos combinados. Comercio Exterior, 53(5), 453-465.

Boltvinik, J. y Hernández, E. (1999). Pobreza y distribución del ingreso en México. México: Siglo Xxi editores.

Caloca, O. R. y Ortiz, E. O. (2016). Situación espacial de la pobreza en el Distrito Federal, Milpa Alta 1990-2010. Análisis Económico, XхXI(76), 95-122.

Camberos, M. y Bracamontes, J. (1995). Análisis comparativo de la pobreza en la frontera norte de México. México: Centro de Investigación en Alimentación y Desarrollo.

Comité Técnico para la Medición de la Pobreza (CTMP). (2002). Medición de la pobreza: variantes metodológicas y estimación preliminar (Documentos de Investigación, Vol. 1). México: Sedesol.

Consejo Nacional para la Evaluación de la Política Social (Coneval). (2009). Informe de pobreza multidimensional 2008. México: Autor.

Consejo Nacional para la Evaluación de la Política Social (Coneval). (2010). Metodología para la medición multidimensional de la pobreza en México. México: Autor.

Consejo Nacional para la Evaluación de la Política Social (Coneval). (2011). Pobreza en México y las entidades federativas 2008-2010. México: Autor.

Consejo Nacional para la Evaluación de la Política Social (Coneval). (2017). Informe de la evolución de la pobreza 2010-2016. México: Autor.

Cortés, F., Benegas, I., Fernández, T. y Mora, M. (2007). Perfiles de la pobreza en Chiapas. Sociológica, 22(63), 19-50.

Damián, A. (2009). La pobreza, los hogares y la ocupación en el DF en 2004, El Colegio de México y el Consejo de Evaluación del Desarrollo Social en el Distrito Federal. (Informe de Investigación). México: Consejo de Evaluación del Desarrollo Social del DF, El Colegio de México.

Feres, J. y Mancero, X. (2000). El método de las necesidades básicas insatisfechas (NBI) y sus aplicaciones en América Latina. En Taller 5: La medición de la pobreza: métodos y aplicaciones. Programa para el mejoramiento de las encuestas y la medición de las condiciones de vida en América Latina y el Caribe (Mecovi). CEPAL.

Fuentes, C. M. (2009). La estructura espacial urbana y accesibilidad diferenciada a centros de empleo en Ciudad Juárez, Chihuahua. Región y Sociedad, xx(44), 117144.

Fuentes, C. M. y Peña, S. (2010). Globalization and its Effects on the Urban Socio-Spatial Structure of a Transfrontier Metropolis: El Paso, Tx.-Ciudad Juárez, Chih.-Sunland Park, NM. En K. Staudt, C. Fuentes y J. Monárrez (Eds.), Cities and Citizenship at the U.S.-Mexico Border: The Paso del Norte Metropolitan Region (pp. 93-117). Nueva York, Estados Unidos de América: Palgrave Macmillan.

Fuentes, C. M. y Peña, S. (2017). Las fronteras de México: Nodos del sistema global de las drogas prohibidas. México: Flacso, IDRC, CRDI, El Colef. 
Fuentes, C. M., Peña, S. y Hernández, V. (2012). La geografía de la pobreza urbana en Ciudad Juárez, Chihuahua: Dinámica y evolución. México: El Colef, Conacyt, Fomix, Gobierno Municipal de Juárez.

Garza-Rodríguez, J. (2016). Los determinantes de la pobreza en los estados mexicanos en la frontera con Estados Unidos. Estudios Fronterizos, 17(33), 1-19.

González, M. (1986). Los recursos de la pobreza. Familias de bajos ingresos en Guadalajara. México: El Colegio de Jalisco, ciesas.

Gordon, D. (2006). The Concept and Measurement of Poverty. En C. Pantazis, D. Gordon y R. Levitas (Eds.), Poverty and Social Exclusion in Britain (pp. 28-69). Gran Bretaña, Reino Unido: The Millennium Survey, Bristol, The Policy Press.

Instituto Nacional de Estadística, Geografía e Informática (Inegi). (1990). Censo de Población y Vivienda. México: Autor.

Instituto Nacional de Estadística, Geografía e Informática (Inegi). (2000). XII Censo de Población y Vivienda. México: Autor.

Instituto Nacional de Estadística, Geografía e Informática (Inegi). (2010). XII Censo de Población y Vivienda. México: Autor.

Instituto Nacional de Estadística, Geografía e Informática (Inegi). (2011). Metodología para la medición multidimensional de la pobreza en México. Revista Internacional de Estadística y Geografía, 2(1), 33-48.

Limas, M. (2010). Geografía de la pobreza en Ciudad Juárez: una perspectiva de género. México: Universidad Autónoma de Ciudad Juárez.

Monárrez, J. y Cervera, L. (2013). Spatial and temporal behavior of three paradigmatic cases of violence in Ciudad Juarez, Chihuahua Mexico: Feminicide, homicide and involuntary disappearances of girls and women (1993-2013). México: El Colegio de la Frontera Norte.

Sánchez, A., Boltvinik, J., Ruiz, F. y Figueroa, H. (2011). Índice de desarrollo social de las unidades territoriales del Distrito Federal delegación, colonia y manzana. México: Evalúa DF, Consejo de Evaluación del Desarrollo Social del Distrito Federal.

\section{Agradecimientos}

Los autores agradecen el apoyo financiero del Fondo Mixto Conacyt-Gobierno Municipal de Juárez para la realización de este trabajo. Especial agradecimiento a los revisores anónimos por sus valiosas sugerencias para mejorar el artículo.

César Mario Fuentes

Mexicano. Doctorado en planeación urbana y regional por la Universidad del Sur de California, realizó sus estudios de maestría en desarrollo regional en El Colef. En la actualidad está adscrito al Departamento de Estudios Urbanos y del Medio Ambiente de El Colef. Sus líneas de investigación son: planeación urbana transfronteriza, seguridad urbana y geografía urbana. Entre sus más recientes publicaciones: Fuentes, C. y Peña S. (2017). Las fronteras de México: Nodos del sistema global de las drogas prohibidas. El Colef, Flacso Ecuador e IDRC. 


\title{
Sergio Peña
}

Mexicano. Estudió el doctorado en planeación urbana en la Universidad Estatal de Florida. Trabaja en El Colegio de la Frontera Norte, adscrito al departamento de Estudios Urbanos y del Medio Ambiente. Su tema de investigación se ha enfocado al análisis de las relaciones transfronterizas y la cooperación, con énfasis en los aspectos de planeación urbana y regional. Entre sus más recientes publicaciones: Peña, S. (2016). Teoría, procesos y práctica de la planeación urbana y regional. México: El Colef y Peña, S. (2016). El impuesto predial en Ciudad Juárez desde una perspectiva de equidad. Economía, Sociedad y Territorio, XVI(51), 519-542.

\begin{abstract}
Vladimir Hernández
Mexicano. Doctor en Ciencias Sociales, maestro en Desarrollo Regional por El Colegio de la Frontera Norte y licenciado en Geografía por la Universidad Nacional Autónoma de México. Actualmente es profesor en el departamento de Arquitectura de la Universidad Autónoma de Ciudad Juárez, México, y miembro del Sistema Nacional de Investigadores. Línea de investigación: análisis geoespacial urbano-regional, seguridad vial. Publicaciones recientes: Hernández, V. (Coord.). (2016). Siniestros viales y análisis espacial. Estudio de los accidentes de tránsito con sistemas de información geográfica en Ciudad Juárez, Mexicali y Toluca. Ciudad Juárez, Chihuahua: Universidad Autónoma de Ciudad Juárez.
\end{abstract}

